\title{
Essais
}

ESSAIS

Revue interdisciplinaire d'Humanités

«L'affaire Scala et les anarchistes »: représentations sociales du désordre pendant la transition démocratique espagnole

\section{Arnaud Dolidier}

\section{(2) OpenEdition \\ Journals \\ Édition électronique \\ URL : https://journals.openedition.org/essais/9379 \\ DOI : $10.4000 /$ essais. 9379 \\ ISSN : 2276-0970 \\ Éditeur \\ École doctorale Montaigne Humanités}

\section{Édition imprimée}

Date de publication : 15 mai 2013

Pagination : 139-160

ISBN : 978-2-9544269-0-7

ISSN : 2417-4211

\section{Référence électronique}

Arnaud Dolidier, « «L'affaire Scala et les anarchistes » : représentations sociales du désordre pendant la transition démocratique espagnole », Essais [En ligne], 3 | 2013, mis en ligne le 01 septembre 2021, consulté le 08 septembre 2021. URL : http://journals.openedition.org/essais/9379 ; DOI : https:// doi.org/10.4000/essais.9379 


\section{"L'affaire Scala et les anarchistes": représentations sociales du désordre pendant la transition démocratique espagnole}

\section{Arnaud Dolidier}

Le 15 janvier 1978, la Confédération Nationale du Travail (CNT), centrale anarcho-syndicaliste, convoque une manifestation contre les Pactes de la Moncloa à Barcelone. Signés par les forces politiques de l'opposition (Parti Communiste Espagnol, Parti Socialiste Ouvrier Espagnol) et le gouvernement en octobre 1977, ces pactes sont présentés comme le remède à la crise qui frappe le pays depuis 1973. Il s'agit d'accords économiques et sociaux qui ont pour objectif de normaliser la situation dans les usines et les entreprises, fixer les rapports de force entre patrons et travailleurs afin de mettre un terme à la conflictualité sociale ${ }^{1}$. Ils viennent s'ajouter à une série de mesures ${ }^{2}$ afin de réguler les conflits sociaux à l'aide d'une politique interclassiste, éliminant dans le même temps les interprétations du moment fondées sur la lutte des classes. Bien que les deux syndicats majoritaires (Commissions Ouvrières et Union Générale des Travailleurs) présentent ces accords comme une victoire pour les travailleurs au nom de la "stabilisation démocratique ", ils provoquent dans le même temps la colère à plusieurs endroits. En effet, les pactes prévoient diverses mesures en faveur du patronat ${ }^{3}$, comme le libre licenciement ou le plafonnement des salaires. Hormis la fronde de quelques sections locales des

1 En 1976, 3638957 grévistes sont recensés, pour 110016240 heures de travail perdues. En 1977, il y a 2317026 grévistes pour 92572050 heures de travail perdues. En 1978, ce sont 3633004 grévistes pour 128738478 heures de travail perdues. Ces chiffres s'accroissent en 1979, avec 5752304 grévistes et 171067049 heures de travail perdues, pour connaître une baisse en 1980 avec 2461061 grévistes et 108625662 heures de travail perdues. Sources : Sagardoy J.A., León Blanco D., El poder sindical en España, Barcelona, 1982.

2 Telles que les élections syndicales et les comités d'entreprises. Sur le monde du travail et la « transition syndicale ", voir Fishman Robert M., Organización obrera y retorno a la democracia en España, Madrid, Centro de Investigaciones Sociológicas, 1996, et Kohler H., El movimiento sindical en España. Transición democrática, regionalismo y modernización económica, Madrid, Fundamentos, 1995.

3 Voir Los pactos de la Moncloa, Servicio Central de Publicaciones, Presidencia del Gobierno, Madrid, 1977. 
CC.OO et de l'UGT - très vite rappelées à l'ordre par leur hiérarchie - et de quelques syndicats et assemblées ouvrières à l'échelle locale, la CNT est la seule organisation nationale à s'y opposer. Face au modèle de négociation et à la politique de pactes qui semble inéluctable, elle impulse une campagne nationale à leur encontre, qui s'ajoute au rejet des élections syndicales et à l'instauration des comités d'entreprise comme nouveau cadre de négociation entre travailleurs et patronat.

En 1976, diverses composantes libertaires s'attellent à reconstruire l'organisation anarcho-syndicaliste historique, afin de peser sur les mouvements sociaux qui, à la même époque, se multiplient dans différentes branches comme dans le bâtiment, la métallurgie, la banque, l'enseignement, les transports, dans diverses usines comme celle d'Intelsa, Motor Ibérica, Roca et bien d'autres. Les conflits dans les quartiers, les grèves générales de 1976 à Vitoria, Sabadell, ou dans le Baix Llobregat, témoignent d'une recrudescence des mobilisations sociales et politiques depuis la mort du dictateur. En effet, le cycle d'action collective protestataire, amorcé dans les années 1960, s'intensifie considérablement au début de l'ère postfranquiste. C'est dans un contexte marqué par d'intenses mouvements sociaux que les multiples groupes affinitaires, collectifs, organisations anarchistes diverses, tentent de reconstruire les " institutions " historiques du " Mouvement Libertaire" ». Face à l'intégration des centrales syndicales majoritaires au jeu politique par le biais du pacte social instauré par le gouvernement Suárez, la CNT, en 1977, adopte une stratégie fondée sur l'action directe ${ }^{5}$ et l'assembléisme. Son rejet du pacte social s'accompagne d'autres alternatives, notamment celle qui postule que l'assemblée ouvrière a la capacité de négocier et de décider, au lieu des comités d'entreprises qui instaurent un modèle de syndicalisme de représentation. Les projets anarcho-syndicalistes ne paraissent pas tant marginaux contrairement à ce que laisse penser l'historiographie, puisque la CNT revendique début

4 Les questions autours de l'objet "Mouvement Libertaire " peuvent être sources d'infinies polémiques quand il s'agit de le définir. L’appellation « Mouvement Libertaire » naît pendant la Guerre Civile et se consolide dans l'exil. Elle fait référence à diverses entités officielles qui le structurent et lui donnent sens, au moment où celles-ci subissent tous types de transformations liées aux contingences de la guerre. Nous reprenons ici la définition livrée par l'historien François Godicheau : «En son centre, on trouve la CNT, institution à laquelle sont greffés d'autres groupements comme la FAI, les cadres de défenses confédéraux, les Jeunesses Libertaires, les Mujeres Libres, sans oublier les journaux, les institutions culturelles comme les athénées, etc. », in François Godicheau, "La domestication de la révolution : du viol des principes doctrinaux à la patrimonialisation de l'identité anarchiste en passant par l'intégration de la CNT à l'État Républicain (1936-1939) ", in Marie-Claude Chaput (éd.), De l'anarchisme au courants alternatifs (XIX-XXI' siècle), Regards, $\mathrm{n}^{\circ}$ 9, Publidix, Université de Paris X-Nanterre, 2006, p. 149-173.

5 L'action directe consiste à défendre les intérêts des travailleurs sans l'intermédiaire de l'État ni d'aucune instance arbitrale. Elle se distingue par le refus de toute médiation sociale. Elle peut être violente ou non. 
1977 près de 300000 affiliés $^{6}$, et bénéficie d'une implantation de plus en plus importante à mesure qu'elle s'investit dans les mouvements sociaux qui éclatent ici et là dans le monde du travail et dans les quartiers.

C'est dans ce contexte qu'a lieu la manifestation du 15 janvier 1978 à laquelle participent 10000 personnes. À $13 \mathrm{~h}$, lorsque celle-ci se termine, un groupe de jeunes cénétistes se retrouve devant la salle des fêtes Scala dans le centre de la ville et l'arrose à coup de cocktails Molotov. Le bâtiment prend feu. Quatre salariés qui se trouvaient à l'intérieur sont retrouvés morts ${ }^{7}$. La presse, à l'unanimité, ainsi que l'ensemble des élites politiques et syndicales, condamnent cet attentat. Le 17 janvier, un communiqué de la police repris par les médias affirme avoir arrêté les auteurs de l'incendie, affiliés à la CNT. Sept personnes sont arrêtées à Barcelone, mais la police procède aussi à d'autres mises en détention, une centaine environ, dans les localités de Rubi et de l'Hospitalet. Le communiqué parle d'un commando anarchiste présenté comme le bras armé de la centrale anarcho-syndicaliste. La Fédération Anarchiste Ibérique (FAI) et les Jeunesses Libertaires (JJ.LL) sont décrites comme des organisations paramilitaires au service de celle-ci. L'Association Internationale des Travailleurs (AIT) est présentée comme une organisation terroriste anarchiste internationale. S'en suit une campagne de marginalisation et de discrédit, de criminalisation et de diffamation qui se traduit par une perte d'adhérents et par la recrudescence des tensions internes au sein de la CNT.

Les historiens qui se sont penchés sur l'affaire Scala ont mis en évidence l'idée d'une attaque conçue et pilotée par les instances de l'État pour déstabiliser le mouvement libertaire dans son ensemble, et la CNT en particulier. C'est l'explication qu'apporte l'historien Bernat Muniesa ${ }^{8}$, mais également Juan Alcalde ${ }^{9}$ ou encore José Luis Gutiérrez Molina ${ }^{10}$. Ces travaux reprennent l'idée du complot avancée par les instances officielles de la CNT de l'époque et

6 Ce chiffre est avancé par Pablo César Carmona Pascual, dans, Transiciones. De la asamblea obrera al proceso de pacto social. CNT (1976-1981), Fundación de Estudios Libertarios Anselmo Lorenzo, Madrid, 2004, p. 7. Dans le même ouvrage, l'auteur affirme qu'en septembre 1977 la CNT compte 116900 affiliés, pour 30288 en décembre 1979, p. 115. Ces dernières estimations semblent plus près de la réalité, compte tenu des tensions internes qui ont participé à une perte croissante d'adhérents. À titre comparatif, en juillet 1977, Nicolas Redondo, leader de l'UGT, affirme que l'organisation syndicale socialiste a distribué 700000 carnets et revendique un nombre de 500000 adhérents. Les CC.OO revendiquent pour leur part 1300000 adhérents début septembre 1977.

7 Deux des quatre travailleurs appartenaient à la CNT.

8 Muniesa Bernat, Dictadura y monarquía en España: de 1939 hasta la actualidad, Barcelona, Ariel, 1996.

9 Alcalde Juan José, Los servicios secretos en España. La represión contra el movimiento libertario español (1939-1995), Madrid, Edición del autor, 1996.

10 Gutiérrez Molina José Luis, «El caso Scala de Barcelona », in El Estado frente a la anarquía: los grandes procesos contra el anarquismo español: 1883-1982, Madrid, Síntesis, 2008, p. 345-371. 
mettent en évidence le fait qu'avec son rejet du pacte social, celle-ci représentait un danger pour le gouvernement qui recherchait la stabilité et tentait de mettre fin aux mobilisations ouvrières. L'idée du complot s'appuie sur les enquêtes internes effectuées par le syndicat, qui mettent à jour des indices prouvant les infiltrations policières au sein de ses structures. Joaquin Gambin ${ }^{11}$ est un indicateur de la police, recruté par la Brigade d'Information (ancienne Brigade politicosociale) et par sa cellule anti-anarchiste dirigée par José Maria Escudero Tejada ${ }^{12}$. Cet indicateur est déjà à l'origine de l'arrestation de 54 militants en janvier 1977 qui voulaient reconstruire la FAI. Volatilisé, on le retrouve à Barcelone en janvier 1978. Après avoir infiltré un groupe de jeunes cénétistes ${ }^{13}$, il les incite à commettre une action violente, pour disparaître à nouveau. Par ailleurs, l'enquête de la CNT et de ses avocats montre que l'incendie du bâtiment n'a pas pu être causé par de simples cocktails molotovs. De manière générale, le procès est parsemé d'incohérences et d'irrégularités ${ }^{14}$. Il aboutit, en 1980, à la condamnation de cinq militants à plusieurs années de prison ${ }^{15}$.

L'historiographie s'est penchée sur l'affaire Scala pour mettre en évidence ses conséquences sur le devenir de l'anarchisme espagnol. Pourtant, cet événement a eu des répercussions bien au-delà du monde libertaire, dans la mesure où il s'inscrit dans la consolidation de la culture politique démocratique dominante des Espagnols, participant à la stabilisation d'un cadre d'identification collective nationale. En effet, les discours dominants ont produit un récit de l'événement dans lequel des sujets ont été re-signifiés pour être agencés au sein d'une représentation globale de "la société démocratique ». Pour que le complot puisse produire les effets escomptés - à savoir la marginalisation des

11 Sur le parcours de l'indicateur de la police, Gambín, voir Gutierrez Molina, José Luis, ibid., p. 357-360.

12 Il s'agit de l'un des chefs directs de Gambin. Mais on peut aussi citer l'inspecteur de police José Gregorio Lopez Marin, qui prends l'initiative de ficher et de faire infiltrer Gambin au sein du mouvement libertaire. Dans cette affaire, le commissaire de la brigade d'information, Conesa Escudero, ainsi que le ministre de l'intérieur, Martin Villa, sont aussi impliqués. Voir Juan Alcalde, op. cit.

13 L'un de ces jeunes cénétistes a écrit, entre autres, un récit sur l'épisode Scala. Il évoque notamment la rencontre, lui et ses camarades, avec l'indicateur de la police. Voir Cañadas Gascón Xavier, El caso Scala. Terrorismo de Estado y algo más, Barcelona, Virus editorial, 2008.

14 Pour Juan Alcalde, l'affaire Scala est le produit de relations opaques entre responsables politiques, fonctionnaires, militaires et militants d'extrême droite. Celles-ci s'inscrivent dans la "stratégie de la tension ", c'est-à-dire, dans la préparation d'actions contre-insurrectionnelles pour justifier l'écrasement de mouvements révolutionnaires ou de mouvements sociaux. Sur la stratégie de la tension, voir Laurent Frédéric, L'orchestre noir, Stock, 1978. Pour le cas espagnol, lire le chapitre XIII de la troisième partie de la thèse de Sophie Baby, op. cit., intitulé, "Des "incontrôlés" aux GAL : la "guerre sale" et le terrorisme d'État ", p. 572-624.

15 José Cuevas, Xavier Cañadas et Arturo Palma écopent de dix-sept ans de prison pour homicide volontaire, Luis Muñoz à deux ans et six mois pour complicité et Rosa López à cinq mois de prison pour rétention d'informations. 
forces libertaires - encore faut-il qu'il existe une série de catégories disponibles pour véhiculer des valeurs, des principes et ainsi alimenter des représentations sociales spécifiques dans lesquelles les mots d'anarchisme et d'anarchiste renvoient à des images de désordre, de violence, de peur et de menace.

De manière simple, ce travail, à partir de l'analyse de "l'affaire Scala ", pose la question de savoir comment le concept de "société " est utilisé dans les discours publics et quels en sont ses effets. L'objectif est de comprendre de quelle manière le terme participe à la construction d'un sens commun qui légitime un nouvel ordre social, fondé sur un jeu spéculaire entre sujets politiques citoyen et anarchiste. Car le terme de "société " participe à la réification de plusieurs groupes sociaux ; il est l'objet de divers usages politiques afin de diffuser une vision globale de relations sociales stabilisées. Ainsi, il est question de montrer quelles sont les mutations qu'ont subies les figures de l'ennemi intérieur au début de l'ère postfranquiste - postulant que les termes terrorisme et terroriste ont été réadaptés au contexte démocratique par le biais de la figure anarchiste. Il s'agit également de démontrer comment le mot de "société ", articulé à d'autres concepts, a rendu possible ces mutations. Par ailleurs, l'objectif final est de s'interroger sur la pertinence heuristique du terme en tant que concept analytique.

Pour répondre à ces questions, ce travail se base sur l'analyse de deux journaux, La Vanguardia de Barcelone, et El $A B C$ de Madrid. Ceux-ci n'ont pas été choisis au hasard : le premier, porte-parole de la bourgeoisie catalane, et le second, relai des monarchistes et des réformistes franquistes, ont en commun la réactualisation de leurs lignes politiques conservatrices pour s'adapter à la réalité " démocratique » du moment. On peut détecter lors de l'affaire Scala une recomposition du discours franquiste, notamment sur les questions de sécurité et de violence. Les articles et les commentaires sur l'événement Scala ont été écrits entre les mois de janvier et février. Ensuite, ces deux quotidiens abandonnent le traitement spectaculaire accordé aux anarchistes, pour écrire des brèves sur le déroulement du procès. Les articles de ces deux journaux sont parfois identiques, reprenant les mêmes commentaires, répondant à la logique du «montage médiatico-policier». Selon le sociologue Mathieu Rigouste, un montage médiatico-policier « fonctionne comme un assemblage de machines de commandement et de spectacle ${ }^{16} »$. Ce concept ne pose pas comme a priori le caractère monolithique du monde de la presse écrite et de la police, mais appréhende leur rôle en tant " qu'appareil d'hégémonie ${ }^{17}$ ». Le concept de

16 Rigouste M., L'ennemi intérieur. La généalogie coloniale et militaire de l'ordre sécuritaire dans la France contemporaine, La Découverte, 2009, p. 310.

17 La notion d'appareil d'hégémonie développée par Antonio Gramsci désigne la part que prennent les institutions dans la consolidation et l'instauration de l'hégémonie. Celle-ci se définit comme le processus par lequel les classes dominantes construisent, légitiment et perpétuent leur domination par l'assentiment et le consentement des classes dominées. Sur la presse comme appareil 
montage médiatico-policier sert à montrer comment s'opère une construction discursive de l'événement à partir de dispositifs de savoirs/pouvoirs sur divers aspects de la réalité. La principale source utilisée par les journaux est le communiqué de police publié le 17 janvier. Plusieurs discours participent ainsi à la résurgence de la figure sociale de l'anarchiste-poseur-de-bombe. Il s'agit de les analyser pour replacer ensuite les interprétations de l'événement dans les débats publics de l'époque, notamment ceux sur la sécurité.

\section{La réactivation d'une figure de l'ennemi intérieur : l'anarchiste- poseur-de-bombe}

Les mouvements sociaux qui caractérisent les débuts de l'ère postfranquiste s'accompagnent d'un cycle de violence politique sans précédent, qui contraste avec le mythe couramment répandu d'une " transition-modèle " pacifique $^{18}$. Aux violences des groupuscules révolutionnaires d'extrême gauche $\left(\mathrm{GRAPO}^{19}\right.$, FRAP $\left.{ }^{20}\right)$, s'ajoute la terreur orchestrée par les nostalgiques de l'ancien régime (BVE ${ }^{21}$, "Guérilleros du Christ Roi »), ainsi que le maintien des structures répressives franquistes. L'instauration du jeu démocratique parlementaire moderne et l'évolution des réformes se sont traduites par l'intégration institutionnelle de nombreuses composantes politiques ayant renoncé à la rupture antifranquiste, mais ont conduit dans le même temps à un regain de violences politiques chez ceux qui refusaient la manière dont le processus transitionnel était mené.

Ces violences sont au centre des discours publics et font l'objet d'interprétations qui réactivent des peurs solidement ancrées dans les imaginaires collectifs : celles d'une révolution bolchevique, d'un coup d'État militaire ou d'une nouvelle guerre civile ${ }^{22}$. La résurgence de ces peurs coïncide avec une série d'attentats, à commencer par l'un des plus violents d'entre eux, celui de la " semaine noire » en janvier 1977 à Madrid dans la gare d'Atocha, qui se solde par dix morts et deux prises d'otages ${ }^{23}$. L'évocation des peurs et des incerti-

d'hégémonie privé, voir Tosel André, «La presse comme appareil d'hégémonie dans les cahiers de prison ", in Le marxisme du XXe siècle, Paris, Syllepse, 2009. Pour une présentation des théories gramsciennes, voir Gramsci Antonio, Guerre de mouvement et guerre de position. Textes choisis et présentés par Razmig Keucheyan, Paris, La Fabrique, 2011.

18 Sur le mythe de la "transition-modèle ", voir André-Bazzana Bénédicte, Mitos y mentiras de la transición, Barcelone, El viejo topo, 2006.

19 Grupos de Resistencia Antifascista del Primero de Octubre.

20 Frente Revolucionario Antifascista y Patriota.

21 Batallón Vasco Espańol.

22 Sur la Guerre Civile espagnole dans l'imaginaire collectif pendant la transition, voir Aguilar Fernández Paloma, Memoria y olvido de la Guerra Civil español, Madrid, Alianza Ed., 1996.

23 Sur l'attentat d'Atocha, voir l'article de Baby Sophie, «Violence et transition en Espagne : La 
tudes, l'interprétation des violences politiques comme tentatives de déstabiliser la démocratie naissante, jettent les bases d'une culture politique démocratique fondée sur la « réconciliation nationale » entre les citoyens. Dans ces discours, ce ne sont pas certaines formes de violences politiques qui sont condamnées, mais la violence en tant que telle ${ }^{24}$, hormis celle de l'État. En effet, deux ans après la mort du dictateur, le mot violence est re-signifié : à mesure que disparaît la culture politique de l'antifranquisme, absorbée par le consensus national, le mot "violence " ne participe plus à dénoncer celle des institutions. Elle est au contraire rendue invisible alors qu'elle est encore notable ${ }^{25}$. Au début du processus transitionnel, les mutations politiques de l'État se traduisent par la reconfiguration des discours de légitimation, produisant de nouvelles altérités politiques. La construction de sujets "non-citoyens ", dont les images sont à la fois imprécises et mouvantes, naît à partir d'une instrumentalisation politique de la violence qui conduit à délégitimer certaines ressources du répertoire de l'action collective. On assiste à une substitution de la figure du " rouge " car celle-ci n'est plus opératoire. De plus, l'utilisation du terme de terroriste est chargée négativement à cause de son utilisation sous le franquisme. C'est pourquoi la catégorie " anarchiste » est réactivée. Le terme prend corps dans l'imaginaire collectif au moment de "l'affaire Scala ». Il fait sens et devient un moyen de remplacer et de réadapter l'imaginaire de la figure terroriste au travers d'une articulation entre violence politique et action sociale. Ainsi, le discours de délégitimation de la violence passe par la démonstration que celle de l'État est un fait du passé. Ce discours est rendu possible par une publicité différente faite entre divers attentats et la violence policière.

L'incendie de cette salle des fêtes intervient dans un climat de violence diffuse et de mobilisations collectives, quelques mois après la signature des pactes de la Moncloa. Deux jours après l'attentat, date où paraît le communiqué de la police, les journaux commencent à évoquer l'anarchisme au travers de références historiques. Ainsi La vanguardia publie un article signé par le journaliste Carlos Senti, dans lequel il affirme que :

Barcelone est une ville terriblement sensible aux failles de l'ordre public. Son histoire moderne est tragiquement marquée - et non pas seulement la semaine appelée précisément tragique - par des interruptions de processus démocratiques, qui ont tourné court à cause du désordre et des désillusions qui provoquent chez les gens insécurité et menace. ${ }^{26}$

semaine noire de Madrid (janvier 1977) ", in Dulphy Anne, Léonard Yves (dir.), De la dictature à la démocratie : voix ibériques, Bruxelles, Bern, Berlin, PIE-P, Lang, 2003, p. 85-104.

24 Voir Baby Sophie, Violence et politique dans la transition démocratique espagnole (1975-1982), thèse doctorale, Paris, Université Paris 1 Panthéon-Sorbonne, 2006.

25 Ibid., notamment le chapitre XI de la troisième partie intitulé "Les violences policières ", p. $496-529$.

26 Senti Carlos, "La escalada de la Scala ", La vanguardia, 17/01/1978, p. 19. 
En premier lieu, l'extrait indique que l'auteur s'inscrit dans un régime d'historicité futuriste ${ }^{27}$, où le passé contient les signes annonciateurs du progrès comme unique devenir temporel. Ce progrès, c'est la démocratie. C'est elle qui articule les rapports entre passé, présent et futur et qui donne sens aux expériences du moment. C'est donc un ordre du temps qui suit inéluctablement sa route vers la démocratie. Un temps linéaire, que rien ne peut venir troubler si ce n'est l'acte violent. Celui-ci, perçu comme interruption, est d'autant plus significatif dans ce texte qu'il est illustré à partir de l'évocation de la Semaine Tragique ${ }^{28}$. Cette référence historique et le mot "d'interruption " montrent de quelle façon s'impose dans le récit la naturalisation du concept de "démocratie » et l'idée qu'un processus démocratique est toujours le produit d'une évolution naturelle des sociétés humaines.

Ce passage est révélateur des reconfigurations conceptuelles dans le langage politique, car le vocabulaire de l'ordre, de la sécurité, semble composer avec celui de la démocratie. Dans l'extrait, la démocratie avance inéluctablement contre la violence. C'est un ordre du temps fondé sur la catégorie binaire démocratie/sécurité, qui présuppose l'idée d'une action préventive afin de normaliser le corps social, d'institutionnaliser les phénomènes de violence sociale, d'encadrer et de discipliner le fait contestataire. Les champs lexicaux de l'ordre et de la sécurité, la perception de la menace, ne sont donc plus l'apanage des régimes dictatoriaux, mais s'accordent au langage démocratique. L'auteur ajoute ensuite :

Qui jeta la bombe aux pieds des chevaux du carrosse royal, transformant les noces d'Alphonse XIII et de Victoria en noces de sang ? Le jeune anarchiste Corral, disciple de Ferrer y Guardia, était le fils d'un des fabricants de Sabadell... Nous voudrions éloigner de nos mémoires, y compris héritées, les spectres des violences de rue qui ont toujours mal fini politiquement...29.

27 Conçu par l'historien François Hartog, le concept de régime d'historicité est un outil heuristique qui permet de montrer comment dans chaque présent, le passé et le futur sont représentés. Ce concept propose d'éclairer la tension entre le champ d'expérience et l'horizon d'attente. Comme le rappelle Hartog, le régime d'historicité futuriste est la marque des temps modernes : " La structure temporelle des temps modernes, marquée par l'ouverture du futur et du progrès, est caractérisée par l'asymétrie entre l'expérience et l'attente " (p. 28). Voir Hartog François, Régimes d'historicités, présentismes et expériences du temps, Paris, édition du Seuil, 2003.

28 La Semaine Tragique désigne les événements qui se sont déroulés à Barcelone entre le 26 juillet et le 2 août 1909. Il s'agit au début d'une grève générale appelée par Solidaridad Obrera (organisation syndicale anarchiste, noyau catalan de la future CNT) contre la guerre coloniale au Maroc impulsée par le gouvernement Maura. Cette grève débouche sur une insurrection populaire anticléricale du 26 au 29 juillet, qui sera lourdement réprimée. Les syndicats sont interdits et les écoles laïques sont fermées. Cinq condamnations à mort sont prononcées, dont celle de Franscico Ferrer y Guardia qui, malgré une campagne de solidarité internationale, est exécuté le 13 octobre.

29 Senti Carlos, «La escalada de la Scala », op. cit. 
Ces interruptions dans l'ordre linéaire du temps sont donc le fait des violences de rue. Celles-ci sont rattachées à l'image de l'anarchiste-poseur-debombe. L'anarchiste incarne donc la figure du délinquant politique, susceptible d'aiguiller les " masses " vers des révoltes sociales. L’idée sous-jacente est celle qui dénie à l'anarchisme toute capacité de productions discursives, idéologiques et théoriques. C'est un anarchisme dépolitisé, inscrit dans des narrations où la vision délinquante est hégémonique. Un anarchisme qui ne repose que sur la pratique, selon les préceptes de " la propagande par le fait " conçu à la fin du XIX ${ }^{\mathrm{e}}$ siècle. Cette stratégie politique, rapidement rejetée par les milieux libertaires, a néanmoins servis à produire le mythe de l'anarchiste à la bombe ${ }^{30}$. Celui-ci a permis de contenir les potentialités subversives de l'anarchisme en niant toute intervention théorique de sa part. Il conditionne le corps social à la surveillance d'une menace criminelle qui pourrait frapper à tout moment, comme pendant les années trente.

Dans un contexte transitionnel marqué par la réconciliation nationale, évoquer la Guerre Civile comme argument politique est prohibé de façon implicite au nom du " consensus ». Pourtant, il est permis d'en parler indirectement. Il y a, dans la citation ci-dessus, une référence voilée à la Guerre Civile, contenue dans la formule "acabaron politicamente mal» (" ont mal fini politiquement»). Les violences de rues débouchent sur des fins politiques " tragiques ». Lidée sous-jacente est celle qui propose d'articuler les actions criminelle des anarchistes, concrétisées par des exemples comme la Scala, et l'influence politique que ceux-ci ont pu acquérir à d'autres époques auprès de la population :

En Catalogne l'anarchisme d'autrefois est en train de renaître et peut acquérir des manifestations aussi tragiques que celles d'hier ${ }^{31}$.

La construction discursive de l'événement Scala permet de signaler le danger qui pèse sur la "transition démocratique ". Si l'on ne tient pas compte des activités développées dans le présent par les groupes libertaires, ceux-ci peuvent redevenir majoritaires, comme l'était l'anarchisme d'antan à Barcelone. La menace mise en évidence par les journalistes est celle d'une force invisible qui, à tout moment, peut retrouver une assise populaire majoritaire. La réactivation de la figure du terroriste/anarchiste a pour fondement l'imaginaire du conflit fratricide. Il y a l'idée qu'il faille contenir, isoler, "immuniser " la population contre l'imprégnation des influences libertaires qui auraient pour conséquence une inversion du processus transitionnel tel qu'il est en train de se mener. La mise en évidence des dangers d'une massification des pratiques libertaires et la dépolitisation de ces dernières par le biais du mythe de l'anarchiste-poseur-debombe, révèle une contradiction. En effet, comment des pratiques peuvent-elles se massifier s'il n'y a pas de discours qui les fondent et les légitiment ? Ce qui se

30 Eisenzweig Uri, Les fictions de l'anarchisme, Paris, C. Bourgeois, 2001, p. 19.

31 "Vulgares asesinos", El noticiero universal, 17/01/1978, p. 1. 
joue à travers la réactivation de la figure de l'anarchiste à la bombe, est l'aveu d'impuissance des classes dominantes face à l'émergence de nouveaux discours et formes d'entendements au sein de la population. Pour pallier cela, la mise en scène narrative de l'affaire Scala est guidée par le registre moral :

La bombe contre Fauchon à Paris et les " cocktails Molotov " qui ont détruit l'édifice Scala à Barcelone ont été l'œuvre d'une même main [...] La terreur n'est plus ni de droite ni de gauche, révolutionnaire ou réactionnaire. Elle est seulement cela : terreur. Et bien plus terrorisante que la terreur elle-même est la compréhension de l'incapacité à l'éradiquer, car, l'union morale de l'humanité détruite, toute autre union, quelque soit l'urgence de ses finalités, est impossible ${ }^{32}$.

La figure de l'anarchiste s'inscrit dans l'imaginaire de la terreur et perd ainsi toute crédibilité quant à son intervention sociale et politique. La dépolitisation du récit et le recours au registre moral participe à la réactivation du couple anarchiste/terroriste. La production de cette figure s'inscrit dans une volonté politique : celle d'investir les territoires de l'action sociale pour l'inonder du discours de la menace et de la peur, mais surtout celui de la prévention.

\section{Les mouvements libertaires : entre action sociale et terrorisme}

Dans l'article de Carlos Senti précédemment cité, l'évocation de Ferrer y Guardia indique l'ambiguïté accordée au phénomène anarchiste et montre la reformulation que celui-ci subit. On lui dénie toute dimension politique moderne en le cantonnant à la criminalité, mais on évoque pourtant le nom d'un intellectuel célèbre de l'anarchisme, comme pour insister sur sa responsabilité face aux actes criminels. Le terroriste était le disciple de l'intellectuel. Les anarchistes terroristes qui ne parlent pas mais agissent ont donc des maîtres à penser et ce sont eux qui portent la responsabilité des explosions ${ }^{33}$. On peut donc supposer qu'indirectement, il s'agit de montrer que derrière l'incendie de la Scala se cachent des théoriciens et autres intellectuels qui transgressent les frontières du nouvel ordre politique démocratique :

Les premières recherches effectuées par les fonctionnaires spécialisés de la Direction Supérieure, et d'autres, par la Direction Générale de la Sécurité basée à Madrid, sont parvenues à la conclusion que l'acte a pu être commis par un " commando " intégré dans la Confédération Nationale du Travail. Il s'agit de militants à la fois de la Fédération Anarchiste Ibérique et des Jeunesses Libertaires. La première organisation a pour objectif le développement d'un mouvement social et révolutionnaire et la pratique du communisme libertaire

32 "La libertad no se hace con bombas ", $E l A B C, 18 / 01 / 1978$, p. 4.

33 C'est la thèse d'Uri Eisenzweig, qui met en évidence l'émergence de la figure de l'intellectuel au sein de la modernité politique, non pas comme procureur à l'image de Zola et du "J'accuse ", mais comme accusé en prenant l'exemple de Toni Negri et des accusations portées contre lui dans le déclenchement des révoltes populaires dans l'Italie de 1977. Voir Eisenzweig Uri, in Les fictions de l'anarchisme, op. cit., p. 14. 
comme chemin vers l'anarchie intégrale, participant à toutes les luttes sociales et économiques de caractère revendicative et révolutionnaire, allant même jusqu’à l'insurrection armée. Elle est constituée par des "groupes spécifiques " et des " individualités " dont le crédo est la violence et le terrorisme, servant d'appui à la Confédération Nationale du Travail, avec laquelle elle est intimement liée, en tant que bras armé ${ }^{34}$.

Dans cet extrait, le récit donne à voir les connexions qui existent entre un monde libertaire invisible, sans représentation dans l'espace public autre que le terrorisme, et celui d'un monde visible, dont la parole sert à fabriquer des attentats. Ainsi, la narration construite à partir de l'enquête policière renferme l'idée que le complot anarchiste a été pensé et prémédité depuis les organisations officielles de ce courant politique. Cet aspect indique une reformulation de la fiction anarchiste-poseur-de-bombe. Reformulation, dans le sens où l'anarchisme espagnol bénéficie d'une représentation politique. Derrière les organisations, il y a l'idée que celles-ci cachent en leur sein un réservoir de terroristes prêts à commettre des attentats comme celui de la Scala. L'idée du commando anarchiste, et la manière dont celui-ci est figuré dans le récit journalistique, indiquent que les instances officielles des organisations libertaires ne sont que des façades au service de l'action terroriste. On constate également dans le discours dominant l'inquiétude face à la question de l'insurrection armée : celle-ci est l'une des modalités d'action des mouvements révolutionnaires qui éclatent ici et là à la même époque. Dans les discours dominants, son évocation permet d'alimenter le fantasme d'une " conspiration anarchiste internationale ". Ainsi, le discours opère un brouillage entre action terroriste et contestation sociale promue par la CNT. Deux modalités d'action différentes prises comme un tout, agencées dans un dispositif narratif policier et judiciaire, qui réifie l'ensemble des composantes libertaires pour les présenter comme conspiration au service de "l'anarchie intégrale » :

Les poursuites menées contre les détenus seront transmises aux autorités judiciaires compétentes, avec les restes du matériel inflammable et incendiaire et de nombreux documents de propagande, tracts de la Confédération Nationale du Travail et de la Fédération Anarchiste Ibérique, ainsi que des manuscrits retrouvés également lors des fouilles et qui font référence à ces deux organisations. Les démarches continuent pour identifier et mettre en détention les quatre membres du commando anarchiste, responsable des faits qui sont à l'origine des enquêtes policières ${ }^{35}$.

La façon dont l'enquête policière est mise en intrigue indique que les terroristes bénéficient du soutien d'organisations légales. L'évocation de textes et de tracts appartenant à la CNT et à la FAI sert à montrer qu'aujourd'hui, l'anar-

34 "Un comando anarquista, presunto autor del atentado contra "Scala" ", La vanguardia, 18/01/1978, p. 5. L'article est identique à celui publié le même jour dans $E l A B C$ du 18 janvier, p. 18, avec le titre cette fois "Un comando anarquista, autor del atentado ». 
chiste-poseur-de-bombe, s'il agit comme auparavant à travers l'action violente, bénéficie de la légitimité des structures du "Mouvement Libertaire ». C'est encore l'idée que derrière ces organisations se trame un complot anarchiste. L'autre idée est celle qui consiste à affirmer que c'est par leurs productions théoriques que les organisations libertaires "fabriquent " des terroristes. Il s'agit du même type de dispositif qui avait cours à la fin du XIX ${ }^{e}$ siècle lorsque les idées anarchistes étaient réprimées à cause de leurs responsabilités dans des attentats. Le 7 juin 1896, une bombe explose dans les rues de Barcelone lors d'une procession religieuse. La presse ne se contente plus de condamner les criminels mais tous ceux qui professent des idées anarchistes, demandant des mesures pour les persécuter. Le 2 septembre 1896 une loi répressive est approuvée afin de condamner l'anarchisme, comme en France avec les lois "scélérates » trois ans plus tôt. Dès lors, cet anarchisme de la fin du siècle joue un rôle essentiel dans la naissance du concept moderne de terrorisme ${ }^{36}$. En effet, son refus de toute représentation politique sert à construire l'image de réseaux criminels cachés au sein de la population. Pendant la transition, l'attentat de la Scala est l'occasion de faire évoluer le sens attribué à celui-ci pour rendre à nouveau opératoire le couple anarchiste/terroriste. On constate donc que l'évolution du terme " terroriste ", comme le souligne Pau Casanellas dans l'introduction de sa thèse de doctorat, constitue " une opération d'instrumentalisation politique assez évidente, mais aussi une solution d'une douteuse fonctionnalité épistémologique ${ }^{37}$ ». L'évolution du terme renvoie à l'idée précédemment formulée : étant donné que les gouvernants et les diverses fractions des classes dominantes ignorent ou connaissent mal la manière dont les acteurs donnent du sens au contexte politique, ces opérations discursives répondent au besoin de contenir des tentatives de radicalisation politique, en alimentant le mythe d'une nébuleuse anarchiste internationale. Celle-ci coïncide avec le déclin dans les représentations dominantes de la " pieuvre soviétique ", responsable des désordres internationaux ${ }^{38}$.

La reformulation de l'anarchiste-poseur-de-bombe, intimement articulée à l'évocation et à la responsabilité des instances officielles du mouvement libertaire, permet donc de dépolitiser l'ensemble des composantes anarchistes et de les cantonner à leur dimension criminelle, comme l'illustre cet article publié le 5 février 1978 dans La vanguardia:

La Garde Civile de Madrid a asséné un coup dur aux groupes libertaires et anarchistes qui monopolisent l'attention publique depuis que certains de leurs membres ont provoqué - c'est ce que l'on présume - l'incendie de la Scala de Barcelone. Hier dans l'après-midi, les services spéciaux de la Bénémérita ont placé

36 Eisenzweig Uri, Les fictions de l'anarchisme, op. cit., p. 12.

37 Casanellas Pau, Morir matando. El franquismo en crisis ante la violencia politica. 1968-1977, thèse doctorale, Bellaterra (Cerdanyola del Vallès), UAB, 2011, p. 21.

38 Rigouste Mathieu, L'ennemi intérieur, op. cit., p. 162. 
en détention neuf membres présumés de l'organisation libertaire-anarchiste, et ont découvert au même moment une cache dans la zone madrilène de Moratalaz et un chalet dans un village proche de la capitale, situé route de la Corogne. Les enquêteurs soupçonnent également l'existence de deux caches à Madrid, dans lesquelles un abondant matériel de propagande et d'armes peut être retrouvé.

L'article se poursuit en affirmant que :

Ce mouvement libertaire-anarchiste, inspiré de l'ancienne FAI (Fédération Anarchiste Ibérique, très liée à la CNT), ayant comme base la ville de Condal, a été le protagoniste de nombreux attentats : celui de la Scala - qu'on lui attribue -, des explosifs contre la prison Modélo, contre les tribunaux de San Feliu de Llobregat et Granollers, celui de Badalone, l'ancien asile Duran, etc., ainsi que des braquages contre plusieurs banques. Les personnes détenues hier à Madrid sont supposées être celles qui - à côté d'autres actions similaires -ont placé l'explosif dans un train de banlieue il y a de cela quelques semaines ${ }^{39}$.

Le récit de l'événement Scala par les journaux de l'époque est fondé sur une narration policière et judiciaire qui donne l'illusion d'une mise en série des attentats, renforçant leurs aspects traumatisant, spectaculaire et inexplicable. Donner les noms d'organisations anarchistes permet de conjurer la confusion et le non sens que provoque parmi l'opinion publique cette perception de la mise en série. De plus, l'amalgame est fait entre des événements intervenus sous le franquisme et ceux d'aujourd'hui. Ainsi, l'article reprend à son compte une partie de l'argumentaire de la dictature. Se référant aux actions commises par les libertaires d'hier et d'aujourd'hui, le récit passe sous silence l'évolution du contexte politique et voile par conséquent la violence de l'État. Les espaces et les milieux dans lesquels évoluent les libertaires sont perçus comme asymétriques. La figure de l'anarchiste est donc celle qui permet d'exporter la menace terroriste dans les espaces de la contestation sociale qui se sont ouverts après la mort de Franco.

Comme l'a montré Sophie Baby, l'évolution rhétorique pour identifier les ennemis de l'État est rendue possible par l'imbrication des figures du criminel, du délinquant et du militant contestataire ${ }^{40}$. De plus, les travaux produits par le sociologue Mathieu Rigouste sur l'ennemi intérieur en France, montre également comment à partir des années 1970, la fiction de l'ennemi intérieur a été reconfigurée à partir d'un brouillage entre ces trois figures. Cela a permis de mettre en évidence le risque d'une menace à la fois sociale et politique.

39 "Madrid: un grupo anarquista desarticulado por la guardia civil », La vanguardia, 5/02/1978, p. 1.

40 "La perception de l'ennemi de l'État évolue clairement de la figure du dissident politique à celle du terroriste qui a recours à la violence pour défendre ses idéaux, figure réduite à la fin de la période au collaborateur abertzale. L'étude des arrestations de types politiques a bien montré ce basculement. Le délinquant ordinaire, qui s'attaque à l'ordre social et représente la figure commune de la clientèle policière dans un régime pacifié, est ainsi supplanté par le terroriste qui combat l'ordre politique ». Baby Sophie, op. cit., p. 626. 
Un nouvel ennemi émerge alors, le "terroriste international ", qui s'éloigne de l'ancienne figure du rouge de l'intérieur pour s'élargir aux étudiants, syndicalistes, et, de manière générale, vers tous les secteurs qui critiquent et contestent l'ordre établi et la raison d'État ${ }^{41}$. La perception de nouvelles menaces situées à la gauche du Parti Communiste Français (PCF) semble avoir été possible grâce à la complaisance du " parti » face à la répression qu'a connu le mouvement de mai $68^{42}$. Bien que son analyse reste cantonnée aux territoires français, il semble possible de l'appliquer à la péninsule ibérique. En effet, l'intégration institutionnelle du PCE a fait évoluer l'image des nouvelles menaces intérieures vers le nationalisme basque. Cette intégration est donc à l'origine d'une re-sémantisation du terme " terroriste ». Dès lors, l'analyse de l'affaire Scala montre que, dans cette imbrication, il existe un déplacement sémantique sous-jacent de la menace intérieure qui permet de faire le lien entre dimension criminelle, politique et contestataire. La figure de l'anarchiste est celle qui permet de combiner le visage du délinquant qui s'attaque à l'ordre social et celui du terroriste qui combat l'ordre politique. Dès lors, cette figure est réactivée dans les récits dominants afin que le pouvoir politique puisse " coloniser " par le discours les territoires de la mobilisation collective qui contestent le nouvel ordre social en construction. Cet amalgame entre criminalité et action sociale libertaire est illustré dans l'entretien qu'accorde le ministre de l'intérieur Martin Villa à La Vanguardia le $1^{\text {er }}$ février, où il affirme que :

Concernant le terrorisme, nous avons eu à Barcelone quatre événements, dont deux très préparés, que sont les assassinats de José Maria Bulto et Joaquin Viola, et deux autres faits, également terroristes, d'origine distincte : d'un côté celui " d'El Papus ", dans lequel il apparaît que les personnes impliquées appartiennent aux groupes " ultras ", de l'autre celui de la "Scala », dans lequel sont impliqués les mouvements libertaires. Je dirais que de tous ces événements, celui qui me préoccupe le plus, dans cet ordre des choses, c'est ce dernier, parce que les mouvements libertaires qui circulent à Barcelone y trouvent en un sens leur origine, qui constitue toujours une menace pouvant être dangereuses pour la convivialité pacifique $[\ldots]$

Et le ministre d'ajouter :

41 "L'émergence du terrorisme comme acteur international a coïncidé avec le déclin dans les représentations dominantes de l'image de la pieuvre soviétique, dirigeant dans l'ombre la quasi-totalité des désordres internationaux. Une autre image l'a progressivement remplacée, celle de la "nébuleuse ", désignant un supposé réseau international des désordres. Elle ne reposait plus sur l'idée qu'un centre, une source unique, dirigeait la somme des menaces, mais sur l'image d'une constellation d'éléments menaçants [...] Parallèlement, l'ancienne figure du rouge intérieur a été élargie dans la pensée sécuritaire française aux étudiants, aux syndicalistes, aux mouvements pacifistes ou écologistes, squatteurs, blousons noirs et hippies, aux luttes sociales et de l'immigration, ainsi qu'à toute manifestation visant à critiquer les fondements de la raison d'État et de l'ordre politique, économique et social ». Rigouste Mathieu, op. cit., p. 162.

Ibid. 
Ce qui me préoccupe spécialement à Barcelone, c'est cette action des groupes anarchistes, parce que depuis toujours il s'agit d'une action déstabilisatrice pour la convivialité pacifique à Barcelone ${ }^{43}$.

Dans cet entretien, Martin Villa insiste sur le caractère " déstabilisateur " du mouvement libertaire, sur l'image de désordre et de menace que celui-ci représente à ses yeux. Les mots qu'il emploie ne sont pas anodins : le ministre de l'intérieur est un ancien haut fonctionnaire du régime franquiste. Ayant exercé à des postes comme ceux de Secrétaire Général de l'Organisation Syndicale (de 1969 à 1974) ou comme Gouverneur Civil de Barcelone (en 1974), l'utilisation de l'expression " convivialité pacifique " permet de montrer en quoi l'État est nécessaire à la population afin d'assurer sa protection. Ainsi, l'expression sert à masquer les violences institutionnelles et à faire oublier les méthodes des forces de police qui, à cette époque, sont toujours celles qui avaient cours sous le régime franquiste. De plus, l'opposition des expressions " action déstabilisatrice » et " convivialité pacifique " pose les bases de la réconciliation nationale. Fondée sur la continuité de l'État, celle-ci évince toute responsabilité du personnel politique héritier du franquisme, confrontée " depuis toujours » à la violence anarchiste. Dès lors, ce type de discours élabore des référents à partir d'idéaux et de principes moraux qui permettent de classer les sujets politiques non-citoyens. Les processus d'exclusion et d'intégration au sein d'un nouveau cadre de relations sociales, à partir d'un registre moral adapté au nouveau contexte politique, permettent d'influencer et de faire évoluer les relations et les échanges entre les individus et l'État, en légitimant l'action de ce dernier.

\section{L'anarchiste contre la « société démocratique »}

Le concept de société est utilisé par les anarchistes pour caractériser une réalité sociale inégalitaire et injuste, hiérarchisée et structurée par des rapports de pouvoir. Le terme est toujours suivi d'adjectifs pour insister sur ses aspects négatifs : société capitaliste, société de classe, société bourgeoise, etc. C'est un mot nécessaire dans la culture politique anarchiste, puisqu'il permet également de proclamer l'avènement d'un monde juste : société communiste libertaire, société d'hommes libres, etc. Ils utilisent aussi l'expression de " contre-société ", signifiant par là que la " société » se traduit par un ensemble de relations sociales inégalitaires.

À l'inverse, l'usage du terme de société dans les discours politiques dominants sert à désigner les nouvelles règles du jeu démocratique, fondé sur le libéralisme, et qui conduit à la conversion des anciens sujets à la condition de citoyens. "Société " a donc un usage extensif, dont le sens permet d'inclure la variété de relations sociales et d'échanges existants entre les individus. Les

43 «Entrevista con el ministro del interior Martin Villa », La Vanguardia, 1/02/1978, p. 5-6. Souligné par moi. 
sujets politiques sont renvoyés à une identification citoyenne et marchande et non plus à celle de la classe ou du peuple. Dès lors, le terme de société est articulé à un ordre social qui rejette les postures radicales au profit de la modération, de la négociation et de la réconciliation. Il permet de diffuser une série de référents moraux qui légitime le nouveau gouvernement :

L'universalité du mal ne doit pas être le motif pour renoncer à la défense de la société dans laquelle nous vivons. La violence et la lamentable répétition des attentats ne doivent pas non plus nous conduire à un autre abandon : celui de nos devoirs de démocrates pour demander aux forces engagées des solutions $[\ldots]$. Face à cette situation nous devons exiger l'efficacité du gouvernement. Nous devons produire des mécanismes d'anticipation pour éviter dans la mesure du possible la surprise, et nous devons réclamer la santé morale grâce à laquelle la justice peut suivre avec une sérénité et un acharnement inexorable la capture des coupables. Continuer à admettre que les incendiaires de la Scala et les autres terroristes sont des héros n'est autre que de la lâcheté morale. Travaillons pour une société plus libre et plus juste ${ }^{44}[\ldots]$

Les événements qui font l'objet d'interprétations où les registres de la peur et de la menace sont mobilisés s'accompagnent des termes de "société " et de " citoyens " pour insister sur le caractère non-citoyen des auteurs d'attentats. Parallèlement, dans ces énoncés, le terme d'anarchie est présent comme synonyme de désordre :

Nous réitérons ces mots pour affirmer aujourd'hui, dans une plénitude absolue, l'urgente nécessité qu'a aujourd'hui la démocratie espagnole d'imposer avec rigueur le principe d'autorité dans chaque milieu. Et d'imposer, partout, l'ordre qui émane de la véritable application des lois et de la convivialité pacifique rassurante que génère l'ordre public respecté, en faveur d'une citoyenneté en général et de personnes individuelles en particulier. [...] La démocratie n'est viable pour personne lorsqu' elle est définie comme anarchie publique $[\ldots]^{45}$

On trouve dans cet extrait la rhétorique habituelle du franquisme (imposer le principe d'autorité), contenue dans des journaux de droite, qui ont su adapter leurs discours à la réalité politique démocratique. Ces mots contiennent également les significations morales à travers lesquelles un nouvel ordre social prend corps dans l'imaginaire collectif. Un imaginaire fondé sur la représentation des classes moyennes qui sont en mesure de dépasser les affrontements politiques liés à la lutte des classes. De plus, là encore, on assiste à la continuité du discours franquiste sur la réussite économique du régime et sur sa capacité à faire émerger ces classes moyennes :

Les classes installées hier dans le monde des travailleurs étaient, en toutes conséquences, dans les partis socialistes-marxistes. La majorité de ces masses sont aujourd'hui intégrées aux schémas bourgeois et constituent l'immense classe moyenne du pays ; ceci est une réalité indéniable, une des plus évidentes

44 "La libertad no se hace con bombas ", El ABC, op. cit.

45 "Un pacto contra la violencia y el desorden ", $E l A B C, 14 / 01 / 1978$, p. 2. 
parmi celles qui exercent une influence sur nos avatars politiques [...] Nous devons être à la veille d'un grand pacte entre les secteurs qui constituent la nouvelle bourgeoisie, celle de l'impulsion créatrice et modernisatrice. Pour cela il s'agit d'organiser et de promouvoir une action citoyenne ${ }^{46}$.

Comme l'a montré l'historien Pablo Sánchez León ${ }^{47}$, cet imaginaire est le garant des valeurs morales et des principes démocratiques qui impulsent un nouveau cadre d'identification collective à travers la citoyenneté. Dans le même temps, la construction de ces classes moyennes absorbe les identités de classes, conduisant à l'exclusion dans le champ des représentations de certaines pratiques sociales :

Que dirait le ministre de l'intérieur responsable de l'ordre public aux gens en général, à la société espagnole, concernant la grande inquiétude dans laquelle elle vit ? M.V. : Je dirais premièrement que nous sommes dans une situation préoccupante. Certains chiffres, dans des catégories déterminées de délits contre la propriété, peuvent réellement faire peur, les "braquages de banques " par exemple [...] Nous avons une situation faite d'une certaine insécurité, mais nous avons des villes plus sûres que celles des pays qui nous entourent, et je crois aussi que cela est un bien que non seulement nous devons conserver, mais que nous devons également accroître par l'action du gouvernement, par l'action de la police, et, je crois, par l'action de toutes les forces sociales ${ }^{48}$.

Ainsi, l'intégration conceptuelle des divers aspects de la réalité sociale grâce aux termes de "démocratie ", " citoyenneté " et "société ", s'accompagne de l'institutionnalisation des conflits politiques, par le biais de l'effacement des identités de classes au profit d'un usage toujours plus varié et extensif du terme de citoyenneté : citoyenneté sociale, économique, etc ${ }^{49}$. Les classes moyennes, comme identité chargée d'un capital symbolique et de valeurs morales spécifiques (réconciliation, pacifisme, paix sociale, etc.), participent au rejet de certaines pratiques sociales guidées par la confrontation de classe.

En effet, contenues par un langage démocratique naturalisé, les pratiques sociales comme le sabotage ou les piquets de grève, inscrites auparavant dans une conception antifranquiste, sont à présent associées aux représentations sécuritaires qui guident le nouvel ordre démocratique. L'épisode de la transition se caractérise ainsi par une " transaction conceptuelle ${ }^{50}$ » entre les diverses composantes politiques qui s'accordent sur les significations à attribuer à

46 "La acción ciudadana ", El ABC, 24/01/1978, p. 19.

47 Sánchez León Pablo, «Desclasamiento y desencanto. Representaciones de clase media y poética de la participación democrática en la transición política ", article inédit non publié.

48 "Binomio seguridad-libertad ", in " Entrevista con el ministro del interior Martin Villa ", La Vanguardia, op. cit., p. 6.

49 Pérez Ledesma Manuel, "Ciudadanía », in Javier Fernández Sebastián, Juan Francisco Fuentes (dir.), Diccionario politico y social del siglo XX español, Madrid, Alianza ed., 2008, p. 216-229.

50 Fernández Sebastián Javier, «Democracia », in Diccionario politico y social del siglo XX español, op. cit., p. 345-361. 
des termes comme "démocratie " et détermine le sens du mot "société ", lui-même articulé aux nouvelles représentations de la citoyenneté. Ce sont des concepts interdépendants qui permettent de formuler une vision globale de la réalité :

À l'heure actuelle, la condamnation de l'incendie de la Scala est générale et unanime. Tous les partis politiques sans exception l'ont manifesté. Il faudrait traduire cette condamnation par une action civique et commune entre les forces de l'État, du gouvernement et des partis politiques. Pourquoi, dans ce genre de situation, n'y a-t-il pas également de pactes comme ceux de la Moncloa ? Les terroristes ne parviendront pas à abattre, avec des procédés pareils, un type de société dont ils savent aussi, quand elle leur convient, tirer profit. Ce qu'ils obtiendront, si nous ne trouvons pas une solution, c'est la destruction de la démocratie que nous sommes tous en train d'élaborer. Et pas si mal, certainement ${ }^{51}$.

La menace est une représentation, une construction imaginaire qui prend corps et fait sens au sein de discours politiques qui agencent la réalité sociale à des logiques sécuritaires. La société renvoie à la fois aux valeurs de paix dont les classes moyennes sont les représentantes, mais devient également effective lorsqu'elle est articulée aux représentations de la menace. La peur, comme machine politique, fonctionne dès lors qu'elle est articulée à cette construction imaginaire de "la société " : en témoigne l'invention d'un nouveau concept, celui de la "sécurité citoyenne».

Le terme de "sécurité citoyenne ", amplement repris par la presse écrite, est très rapidement employé dans le langage courant sans être questionné. Cela est en partie lié à la charge négative de l'expression " ordre public » qui renvoie au système franquiste. C'est une formule intimement liée aux représentations libérales de "la société démocratique ", où les individus exercent leurs droits et leurs libertés dans un cadre de défense, de sécurité et de prévention de la menace. Certaines études scientifiques ont mis en évidence que le concept, produit par la droite, n'a pas été combattu par l'opposition ${ }^{52}$. Car dans la presse écrite, mais aussi dans les discours politiques, le récit qui apparait en filigrane est celui qui insiste sur le caractère instable et menaçant du régime démocratique et partant, permet de revaloriser et de reformuler la propagande du système franquiste comme système de stabilité et de paix sociale. Pourtant, si ce concept qui

51 Senti Carlos, op. cit.

52 Voir par exemple, Alonso Pérez F., Seguridad ciudadana, Madrid, Marcial Pons, 1994, ou encore Hurtado Martínez, María del Carmen, Concepto y causas de la inseguridad ciudadana, Cuenca, Universidad de Cuenca, 1999, et l'article de Medina Juanjo, "Inseguridad ciudadana, miedo al delito y policía en Espańa”, Revista electrónica de Ciencia Penal y Criminología. 2003, n 05-03. Les deux premiers ouvrages tentent de définir le concept, et de voir ce qu'il implique dans la formation d'un nouveau cadre de relations citoyennes. L'article insiste sur son caractère ambivalent et met en avant des causes sociales dans la montée de l'insécurité. Pourtant, aucun de ces travaux ne questionnent le concept ni ses effets. Ils le prennent comme point de départ de l'analyse, sans s'interroger sur son « invention». 
donne lieu à une loi n'a pas été questionné ni même contestée par les milieux de l'opposition parlementaire, n'est-ce pas parce qu'un groupe social, en l'occurrence l'élite politique, a dû reformuler de nouvelles fictions du lien social pour garantir une certaine hégémonie ? La " sécurité citoyenne " n’inaugure t-elle pas une nouvelle conception théorique du contrôle sécuritaire, non pas au sein d'un parti ou d'une organisation en particulier, mais dans la " pensée d'État " en général, afin de dépolitiser les délits politiques pour mieux les criminaliser ?

Si l'on part du constat que l'articulation des concepts de société-démocratiecitoyenneté, instaure de nouvelles représentations du lien social et de la menace, la figure de l'anarchiste est centrale dans les législations sur la sécurité citoyenne. L'insécurité est associée à des gestes que les libertaires n'ont de cesse de politiser. En premier lieu, les attaques contre la propriété, mais également la lutte des prisonniers sociaux (presos sociales), des comités de soutien à la Coordination des Prisonniers Espagnols en Luttes (COPEL), par exemple. Les nouvelles menaces mises sur le devant de la scène par la loi sur la Sécurité Citoyenne ne sont pas des phénomènes nouveaux : il s'agit de faits en série qui auparavant faisaient l'objet, notamment dans l'opposition, d'une lecture sociale, conséquence de la misère, du chômage, etc. nourris par des principes politiques comme ceux de l'antifranquisme. En 1978, ils sont interprétés comme la conséquence de l'instauration du cadre politique démocratique qui a pour corolaire l'insécurité. Les classes dominantes inventent de nouvelles lectures criminelles pour légitimer l'instauration du nouvel ordre social et politique. Dès lors, en 1978, l'institution policière tente de se forger une nouvelle légitimité en devenant la garante des libertés citoyennes contre l'insécurité :

Barcelone, autant à cause de sa grande concentration démographique toujours croissante que de son caractère cosmopolite et ouvert à tout type d'influences, est, avec sa zone métropolitaine, une capitale avec un fort indice de criminalité. Cela est dû, entre autres facteurs, au système de vie propre aux grandes villes dans lesquelles abondent les conflits et les tensions, autant au niveau individuel que familial ou social, créant une ambiance qui déshumanise l'individu, protégeant et quelquefois fomentant les conduites et les prédispositions de sujets déterminés à commettre des faits délictueux. Ce qui facilite dans ces circonstances l'anonymat, l'association avec d'autres délinquants et les occasions de tout type qu'offre la société urbaine. Ainsi, avec ce qui vient d'être exposé, on comprendra que beaucoup de délinquants habituels cherchent à Barcelone un refuge idéal pour commettre leurs activités, agissant comme de parfaits parasites, au préjudice de la majorité des citoyens honnêtes. ${ }^{53}$

On retrouve à l'œuvre dans cet extrait les principes théoriques de la conception sécuritaire moderne analysé par Mathieu Rigouste dans la pensée politico-militaire ${ }^{54}$. Dans cet article, il est dit qu'au sein du corps social, au

53 "La policía barcelonesa informa ", La Vanguardia, 16/02/1978, p. 31.

54 Voir la partie II : « la chienlit et les sous développés : la conception du modèle sécuritaire français (1959-1981)», p. 117-181, in Rigouste Mathieu, op. cit. 
milieu des " honnêtes citoyens ", se cachent les " parasites ». On a aussi des formules ambiguës, où l'on sous-entend que le conflit social est un risque d'insécurité. En règle générale, ce texte montre que l'État et sa police se présente comme la "chair » du corps national, mais cherche aussi à décrire comment, chez une partie de la population, la menace prolifère, qu'il faut donc l'immuniser. Ce genre de discours instaure le principe d'autocontrôle de la population, analysé par Michel Foucault dans les sociétés disciplinaires du XVIII et XIX ${ }^{\mathrm{e}}$ siècle $^{55}$. Ainsi, ces énoncés permettent de re-signifier les représentations du territoire social : sous le régime franquiste, il s'agissait de combattre la subversion. Avec l'avènement de l'ordre démocratique, l'espace public est conçu comme un territoire où prolifere la menace. Celle-ci, à la fois sociale et politique, est mise en évidence par la re-configuration des catégories de citoyenneté et d'anarchiste. L'affaire Scala sert donc à discréditer un ensemble de pratiques autrefois interprétées comme antifranquistes. L'événement intervient comme rupture dans la façon d'appréhender les phénomènes sociaux qui surgissent dans la réalité. Au sein d'une société démocratique en transition, il permet le passage dans la morale et le sens commun, d'associations d'idées qui auparavant étaient propres au franquisme. L'affaire Scala devient donc l'occasion pour la police de commencer à se refaire une image neuve, alors même qu'elle n'a pas été épurée.

Ainsi, les mots que nous avons analysés provoquent, selon Javier Fernández Sebastián, "la sensation que le monde moderne ne peut seulement être représenté qu'avec le langage de la modernité, appréhendé comme une structure totale sans échappatoire possible. Le reste n'étant que silence ${ }^{56}$ ". Ils s'inscrivent dans un jeu spéculaire où la société de citoyens fondée sur un imaginaire des classes moyennes participe à la pacification des relations sociales, face à des identités de classes qui renvoient aux " désordres » des conflits sociaux, menaces de violence politique, de délits et d'insécurité mais aussi terrain de jeu du terrorisme. Dans ces configurations sémantiques, l'événement de l'incendie "Scala » fut l'occasion de déplacer les frontières symboliques entre l'action sociale et le terrorisme par le biais de la figure de l'anarchiste-poseur-de-bombe. Cet événement s'inscrit dans l'élaboration de nouvelles représentations sociales du désordre à partir d'un registre moral reformulé et de relations sociales caractérisées par l'enjeu sécuritaire, lui-même agencé à un imaginaire global de " la société ".

\section{Conclusion}

Si le langage n'est pas qu'un moyen de communication mais un réservoir conceptuel permettant de produire des significations de la réalité sociale, alors le terme de "société " renvoie à un imaginaire collectif et à une perception

55 Foucault Michel, Surveiller et punir. Naissance de la prison, Gallimard, Paris, 1975.

56 Fernández Sebastián Javier, Francisco Fuentes Juan (dir.), op. cit., p. 71. 
globale de la réalité construite par les protagonistes d'une époque donnée. Par le biais de séquences narratives au sein desquels des référents moraux guident et régulent les pratiques sociales des individus, la "société " est conçue dans les récits dominants comme le territoire de la pureté, sujet à l'action de terroristes perçus comme des virus proliférant en son sein. À travers l'exemple présenté dans cet article, il s'agissait de montrer que le terme de "société " utilisé par les acteurs de l'époque au moment de l'affaire Scala, était un effet - et non la cause - d'une série spécifiques de représentations sociales du désordre et de la menace, elle-même fondée sur de nouvelles identifications collectives.

Si le terme de "société » reste nécessaire aux sujets pour synthétiser un ensemble de relations sociales, valoriser des identifications collectives ou faire émerger des cultures politiques, en revanche cette notion doit être questionnée quant à sa pertinence heuristique. En effet, "société ", concept fondamental dans la culture moderne, a acquis au fil des siècles la condition d'entité naturelle. Dans le champ de la théorie sociale, elle renvoie à un a priori discursif selon lequel la réalité est découpée entre la sphère des phénomènes matériels, de la "structure sociale ", et celle de la subjectivité, des actions collectives entreprises par les « individus ». L'une des conventions non débattues au sein de l'histoire sociale est justement celle qui présuppose l'existence d'un sens commun chez les acteurs de l'époque, un ensemble de valeurs sociales partagées qui apparaît en filigrane dans l'emploie du terme de "société » et qui a valeur d'injonction. Ainsi, en historicisant les concepts, il est possible d'appréhender la formation des identifications collectives et les multiples expressions de la réalité sociale.

Arnaud Dolidier

Doctorant en études ibériques

EA 3656 AMERIBER

Université Michel de Montaigne Bordeaux 3 arnaud.dolidier@etu.u-bordeaux3.fr

\begin{abstract}
Résumé
Le 15 janvier 1978 à 13h, la salle des fêtes Scala à Barcelone est incendiée, victime d'un attentat terroriste. Deux jours plus tard, la police publie un communiqué dans lequel elle affirme avoir arrêté les coupables, tous faisant partie d'un commando anarchiste. À partir de l'analyse de deux journaux de l'époque, l'objectif de ce travail est de montrer en quoi la production discursive de l'événement a permis de construire des représentations sociales spécifiques de l'anarchisme, et comment celles-ci se sont insérées dans une vision globale de la société. Dès lors, il s'agit de montrer comment les figures sociales de l'ennemi intérieur ont évolué, à mesure que les discours franquistes ont été reconfigurés pour s'adapter à la réalité de l'époque.
\end{abstract}

\title{
Mots-clés
}

Ennemi intérieur, violence, représentations sociales, anarchisme, terrorisme. 


\section{Resumen}

El 15 de enero 1978, a la una de la tarde, la sala de fiesta Scala en Barcelona fue incendiada, víctima de un atentado terrorista. Dos días más tardes, la policía publica un comunicado en el cual afirma haber arrestado los culpables, todos integrados en un comando anarquista. Partiendo del análisis de dos periódicos de la época, este trabajo tiene como objetivo mostrar en qué medida la producción discursiva del acontecimiento permitió construir representaciones sociales especificas del anarquismo, y cómo estas fueron integradas en una visión global de la sociedad. Asi pues, se trata de mostrar cómo las figuras sociales del enemigo interior han cambiado, en la medida que los discursos franquistas fueron reconfigurados para adaptarse a la realidad de la época.

\section{Palabras clave}

Enemigo interior, violencia, representaciones sociales, anarquismo, terrorismo.

\section{Abstract}

On the $15^{\text {th }}$ of January 1978 at 1 p.m., the Barcelona Scala reception Hall was burnt, victim of a terrorist attack. Two days later, the police issued a press release in which they claimed to have arrested the culprits, all part of an anarchist commando. Based on the analysis of two newspapers of that time, the objective of this work is to show how the discursive production of the event has helped build specific social representations of anarchism, and how they are fitted into a global vision of society. Therefore, it comes to show how the social figures of the enemy inside have evolved as nationalist speeches have been readjusted to adapt to the reality of the time.

\section{keywords}

Enemy within, violence, social representations, anarchism, terrorism. 